\title{
Tackling the Climate Conundrum
}

\author{
Dirk von Schneidemesser
}

Review of Greenprint: A New Approach to Cooperation on Climate Change by Aaditya Mattoo and Arvind Subramanian, 2013, Center for Global Development

Many have proposed solutions to the gridlock on international climate change action. Few have put forth convincing arguments that have the added (necessary) benefit of being politically feasible. The suggestions of Aaditya Mattoo and Arvind Subramanian in their recent book Greenprint: A New Approach to Cooperation on Climate Change is an interesting attempt at just that. Greenprint acknowledges important sources of climate policy stagnation and allows these to frame the blue...err...greenprint for the way forward.

It is easy to focus on the obstacles that have been hindering advancement on international climate policy agreements; the focus tends to be on familiar, rather than current or future obstacles. The narrative we hold dear, who is at fault, who can do what, however, may be outdated. Greenprint invites us to update our inventory and focus what is possible in the face of current realities.....as sober and well founded as their argument may be, Mattoo and Subramanian do demand a healthy dose of idealism/optimism. (But let's face it. Without a healthy dose of optimism, you would likely not be reading the book.)

First off, the authors argue that the familiar roles of the North-South divide must be reevaluated. The 'narrative' of historical emissions is not productive, because the global South loses if the North drags its feet, regardless of who is at fault. In short, it is practical and in the interest of both sides to let go of historical emissions; to look forward, not backward.

In the 'New World,' as the authors refer to current global dynamics, 'Dynamic Emerging Economies' (DEEs), are emboldened by their economic growth relative to feeble and indebted industrialized nations, and must take the lead. These DEEs (defined by the authors as Brazil, China, India, and Indonesia) are all large emitters (with members ranking \#1 [China] and \#3 [India] on global emissions) and very vulnerable to climate impacts.

The familiar bargaining structure, with industrialized nations wielding carrots and sticks, is over, and needs to be let go. The recent financial crisis underlined the need to jettison both the 'cash for cuts' ideology and the focus on emissions reductions commitments. Instead, large Southern countries are to eliminate their energy subsidies, prodding the North to apply a sustained price on carbon-raise the stakes through action. This would, the authors claim, result in the private sector kicking off a green technology boom, which would carry us into a 'less undesirable' future ('less undesireable' is a favorite term of Mattoo and Subramanian, indicative of the sober and realistic tone of Greenprint).

The authors review the major trends in discussions on climate policy and equity, and determine that 'preserving development opportunities' is the principle that is most likely to produce an equitable way forward. The point is rightly made that nations must "remain the unit of analysis" because nations sit at the bargaining table. There are significant difficulties in holding an individual accountable for the emissions of their forbearers, which really demonstrates the necessity of moving past the historical emissions frame. Thus, the principle of 'preserving development opportunities' is the most useful in the equity debate, because it tacitly acknowledges the current situation and the history behind it, while keeping the focus on the future.

An important point, which could have been emphasized more strongly, is that in the long term, policies that embody confidence in a greener future will also bring economic benefits associated with green tech and non-energy-intensive sectors. However, the brief mention is understandable since policy is likely to reflect more immediate short to medium term interests. This is evident in the last chapter of Greenprint, which offers an efficient comparison of the consequences of various trade-policy tools in our climate-policy toolbox. The importance of bundling international agreements on climate change with those on trade actions is emphasized, and rightly so, because if left separate, the political leverage of the global North on trade would render the South would be the major loser.

The thoughtful approach to the climate conundrum offered in Greenprint is worthy of consideration. The book combines political realities with moral conscience, offering grounded policy input for those frustrated with the current impasse and the frames that the climate debate has been dragging along with it.

Dirk von Schneidemesser Hertie School of Governance d.schneidemesser@phd.hertie-school.org 